\section{Geology • Geography Dnipro university bulletin}

ISSN 2313-2159 (print) ISSN 2409-9864(online)

Dniprop. Univer.bulletin. Geology, geography., 25(2), 146-150.

Journal home page: geology-dnu-dp.ua

\title{
Определение инфильтрационного питания подземных вод аналитическими и численными методами
}

\author{
Г. П. Евграшкина, Т. П. Мокрицкая, В. К. Марченко
}

Днепровский нацииональный университет имени Олеся Гончара, Днепр, Украина, e-mail:marchenlo_lera@icloud.com

Received 12 October 2017

Received in revised form 01 November 2017

Accepted 10 November 2017

пространстве всех режимообразуюших факторов, в томриторий зависит от точности количественной оценки во времени и фильтрации типа Пуассона и более сложных присутствует в виде свободного постоянного слагаемого и интерпретируется как граничное условие второго рода над уровнем грунтовых вод. В соответствии с известными классификациями его определение относится к научной категории «обратные задачи». Практический опыт ведущих гидрогеологов Украины показывает, что наиболее эффективны методы его определения, в которых исходными данными служат многолетние режимные наблюдения во времени и пространстве. В статье выполнен обзор существующих методов с авторскими примерами их применения при минимуме исходной информации в простых гидрогеологических условиях до относительно сложных, учитывающих фильтрационную анизотропию. Предложены разработки с решением нелинейных уравнений при минимально достаточном количестве исходной информации. Продолжением этих исследований будет решение прямой гидрогеологической задачи «Прогноз уровенного режима» с ориентацией на оползневые участки города Днипро. Во всех видах расчетов в качестве исходных данных использованы режимные наблюдения, выполненные КП «Южукргеология» и изложенные в фондовых отчетных документах, а также гидрогеологические параметры, определенные по результатам откачек. Структура работы выдержана в стиле от простого к сложному.

Ключевые слова: инфильтрачионное питание, обратная гидрогеологическая задача, режимные наблюдения, численные и аналитические методы

\section{Determination of infiltration nutrition of groundwater by analytical and numerical methods}

\author{
G. P. Yevgrashkina, T. P. Mokritskaya, V. K. Marchenko
}

Oles Honchar Dnipro National University, Dnipro, Ukraine, e-mail: marchenlo_lera@icloud.com

Abstract. The definition of infiltration nutrition is an important and compulsory component of the complex prognostic hydrogeological task.Adequacy of mathematical models of changes in hydrogeological conditions of technogenically disturbed territories depends on a number of factors.Namely, from the accuracy of the quantitative evaluation in time and space of all the regimen-forming factors also from infiltration nutrition. This quantity in the Poisson-type and more complex filter equations is present as a free constant term and is interpreted as a boundary condition of the second kind over the groundwater level. In accordance with known classifications, its definition refers to the scientific category "inverse problems". Practical experience of the leading hydrogeologists of Ukraine shows that the most effective methods of its determination, in which the initial data are multiyear observations in time and space. The article reviews the existing methods with author's examples of their application with a minimum of initial information under simple hydrogeological conditions, to relatively complex ones, taking into account the filtration anisotropy. Developments are proposed with the solution of nonlinear equations with a minimal sufficient amount of initial information. The continuation of these studies will be the solution of the direct hydro-geological problem "Prognosis of the level regime" with an orientation toward landslide areas of the city of Dnipro. In all types of calculations, the regime observations made by KP "Yuzhukrgeologia" and outlined in the background report documents, as well as the hydrogeological parameters determined by the results of pumping out, were used as the initial data. The structure of the work is consistent in style from simple to complex. As a result of the conducted researches, it is recommended to build a new regime observational network. The observational network will 
include five wells. Three wells will be located along the current line, and three - across the groundwater flow. Such a network will make it possible to study in greater detail the change in the groundwater table and obtain the most reliable data. All the methods considered are applicable for determiningthe magnitude of infiltration feeding in the study area according to the data of regime observations. After carrying out the research, it can be concluded that the methods described are suitable for solving the stated problems without limitations in the regime observations. The methods presented are in good agreement with each other. This is a confirmation of high reliability. Infiltration nutrition in different years has positive and negative values. In the multi-year section, the value in the sum is positive. This ensures a very slow rise in the water table.

Keywords: infiltration nutrition, reverse hydrogeological problem, regime observations, numerical and analytical methods

Введение. Цель настоящих исследований максимальное использование результатов режимных наблюдений для оценки величины инфильтрационного питания на основе современных методов решения уравнений фильтрации. Для прямого определения инфильтрационного питания используют стационарные установки различных конструкций. Лидером научных достижений этого направления является гидрогеологическая станция «Феофания» НАН Украины (Sitnikov, GolovchenkoJ, Tkachenko, 2003). В нашем регионе в настоящее время такие исследования не проводятся. Недостаток прямых наблюдений - необходимость экстраполяции результатов на большие территории. Преимущества методов, основанных на решении дифференциальных уравнений относительно искомого параметра, заключаются в использовании достаточно большого количества режимных наблюдений во времени и пространстве, что повышает достоверность конечного результата.
Объект исследований - структурный элемент режимной наблюдательной сети города Днипро (рис. 1). Он расположен в Чечеловском районе. Режимные наблюдения выполнены КП «Южукргеология» в период $1983-1995$ годов. Временной интервал наблюдений - 10 суток. Анализ результатов режимных наблюдений позволяет утверждать, что уровенный режим исследуемой территории характеризуется глубоким залеганием (более 5 м от поверхности земли), сезонными колебаниями в пределах 1 м и очень медленным подъёмом в многолетнем режиме (не более 10 см за год). Режимная наблюдательная сеть - это первая и обязательная составляющая современного гидрогеологического мониторинга. Она подлежит плановой модернизации. В ближайшее время её обновления целесообразно создать её структурный элемент, которому соответствует уравнение (10), на основе гидродинамической сетки - 3 скважины по токовой линии и 3 вкрест потока подземных вод.

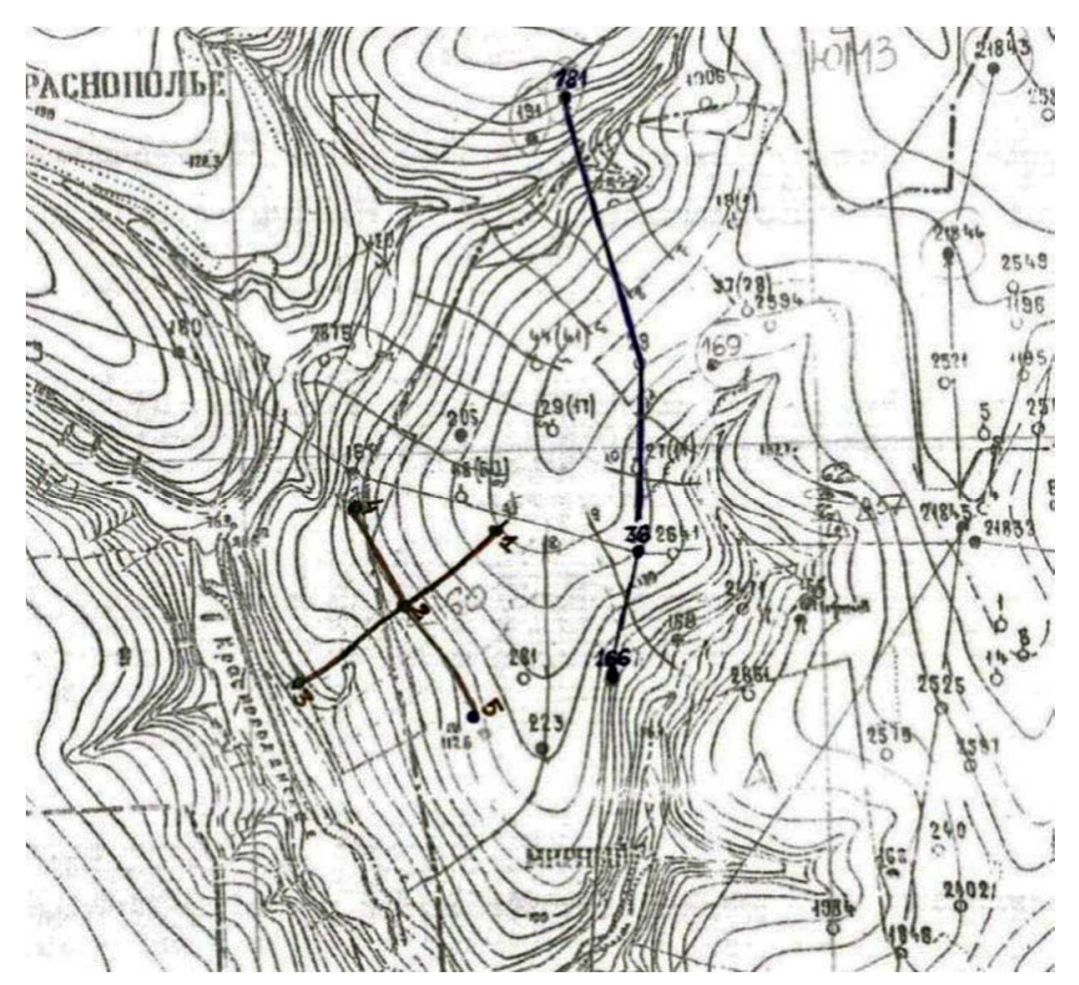

Рис. 1. Гидродинамическая сетка и элементы режимной наблюдательной сети исследуемой территории. М 1: 250000 
Методика расчета величины $\varepsilon$ в каждом конкретном случае определяется количеством исходной информации и геологогидрогеологическими условиями исследуемой территории. Самый простой способ оценки инфильтрационного питания - это нахождение его по формуле предельного подъёма (Zhernov, Soldak, Kushh, Gryza, 1972). Для исследуемой территории верхняя часть разреза, содержащая грунтовые воды, сложена различными суглинками с низкими коэффициентами фильтрации $k$. Этот параметр, определенный КП «Южукргеология» для данного участка методом откачек, изменяется в пределах 0,057-0,19 м/сут. В таких породах, согласно предпосылке Мятиева - Гиринского, движение подземных вод происходит преимущественно по вертикали, горизонтальная составляющая пренебрежимо мала, что и предусмотрено формулой предельного подъёма:

$$
\begin{aligned}
& \Delta h=\frac{\varepsilon t}{\mu}, \\
& \varepsilon=\frac{\Delta h \mu}{t},
\end{aligned}
$$

где $\Delta h-$ величина подъема уровня грунтовых вод за период времени $t$, сут;

$\varepsilon$ - инфильтрационное питание, м/сут;

$\mu$ - коэффициент недостатка водонасыщения, доли единицы;

По формуле (1) выполнены определения величины $\varepsilon$ и сопоставлены с расчетами другими методами. Результаты хорошо согласуются друг с другом. Этот метод относится к аналитическим.

Для оползнеопасных участков в большинстве случаев справедлива расчетная схема «инфильтрация в полуплоскости». Аналитическое решение прямой задачи с использованием этой схемы имеет вид (Rudakov, 1970):

$$
\begin{gathered}
z=\frac{\varepsilon t}{\mu}\left(1-2 i^{2} \operatorname{erfc} \zeta\right), \\
\zeta=\frac{x}{2 \sqrt{2 a t}} .
\end{gathered}
$$

Решение обратной задачи по определению инфильтрационного питания $\varepsilon$ выводим из формулы (2):

$$
\varepsilon=\frac{z \mu}{t\left(1-2 i^{2} e r f c \zeta\right)}
$$

В формулах (2) - (3) приняты следующие обозначения: $z$ - подъем грунтовых вод за период времени $t$, сут;

$\mu$ - коэффициент недостатка насыщения, доли единицы;

$i^{2}$ erfc - табулированная функция (Rudakov, 1970).

Для линейного ряда, состоящего из трех скважин при наличии режимных наблюдений можно использовать конечно-разностное представление уравнения:

$$
\frac{\partial^{2} h}{\partial x^{2}}+\frac{\varepsilon}{t}=\frac{1}{a} \cdot \frac{\partial^{2} h}{\partial t}
$$

в явном виде уравнение имеет вид:

$$
\frac{h_{i-1}^{\tau}-2 h_{i}^{\tau}+h_{i+1}^{\tau}}{(\Delta x)^{2}}+\frac{\varepsilon}{T}=\frac{1}{a} \cdot \frac{h_{i}^{\tau+1}-h_{i}^{\tau}}{\Delta \mathrm{t}},
$$

и по неявной схеме:

$$
\frac{h_{i-1}^{\tau+1}-2 h_{i}^{\tau+1}+h_{i+1}^{\tau+1}}{(\Delta x)^{2}}+\frac{\varepsilon}{T}=\frac{1}{a} \cdot \frac{h_{i}^{\tau+1}-h_{i}^{\tau}}{\Delta \mathrm{t}} .
$$

На исследуемой территории отсутствуют скважины линейного ряда, расположенные на равных расстояниях $\Delta x$, поэтому представляем уравнение (4) по неравномерной схеме с расстояниями между скважинами $\Delta x_{1}$ и $\Delta x_{2}$ :

$$
\left(\frac{h_{i-1}^{\tau}-h_{i}^{\tau}}{\Delta x_{1}}-\frac{h_{i}^{\tau}-h_{i+1}^{\tau}}{\Delta x_{2}}\right) \div \frac{\Delta x_{1}+\Delta x_{2}}{2}=\frac{1}{a} \cdot \frac{h_{i}^{\tau+1}-h_{i}^{\tau}}{\Delta t} .
$$

Аналогично записывается неявная схема. Анализ величин водопроводимости для исследуемой территории свидетельствует о наличии фильтрационной анизотропии. Её учитывает уравнение:

$$
\frac{\partial}{\partial x}\left(T \frac{\partial H}{\partial x}\right) \mp \varepsilon=\mu \frac{\partial H}{\partial t} .
$$

Конечно-разностное его представление имеет вид для неодинаковых расстояний между скважинами:

$$
\begin{gathered}
\left(\frac{T_{i-1}+T_{i}}{2} \cdot \frac{H_{i-1}^{\tau}-H_{i}^{\tau}}{\Delta x_{1}}-\frac{T_{i-1}+T_{i+1}}{2 \Delta x_{2}} \cdot \frac{H_{i}^{\tau}-H_{i+1}^{\tau}}{\Delta x_{2}}\right) \div \\
\frac{\Delta x_{1}+\Delta x_{2}}{2}=\mu \frac{H_{i}^{\tau+1}-H_{i}^{\tau}}{\Delta t} .
\end{gathered}
$$

В уравнениях (4) - (9) приняты следующие обозначения:

$h$ - мощность водонасыщенной части безнапорного водоносного горизонта, м; $x$ - пространственная координата в декартовой системе, м;

$T$ - водопроводимость, ${ }^{2} /$ сут.; 
$a$ - уровнепроводность $\mathrm{M}^{2} /$ сут.;

$t$ - временная координата, сут.;

$i-1, i+1$ - пространственные индексы расчетных точек;

$\tau, \tau+1$ - временные индексы расчетных точек на предыдущий и последующий моменты времени; $\Delta x, \Delta x_{1}, \Delta x_{2}$ - расстояния между расчетными точками или скважинами, м;

$H$ - гидродинамический напор, м.

Остальные обозначения приведены выше.

В. М. Шестаковым (Shestakov, 1979) для учета продольной и поперечной анизотропии предложена гидродинамическая схема «крест». Структурный элемент наблюдательной сети состоит из 5 скважин. Гидрогеологические условия этой схемы описывает двумерное уравнение фильтрации:

$$
\frac{\partial}{\partial x}\left(T \frac{\partial H}{\partial x}\right)+\frac{\partial}{\partial y}\left(T \frac{\partial H}{\partial y}\right)+\varepsilon=\mu \frac{\partial H}{\partial t}
$$

Для такой схемы двумерное уравнение записывается в общем виде следующим образом:

$$
\begin{aligned}
& \frac{\frac{T_{i-1, j}+T_{i, j}}{2} \cdot \frac{H_{i-1, j}^{\tau}-H_{i, j}^{\tau}}{\Delta x}-\frac{T_{i, j}+T_{i+1, j}}{2} \cdot \frac{H_{i, j}^{\tau}-H_{i+1, j}^{\tau}}{\Delta \mathrm{x}}}{\Delta x}+ \\
& \frac{\frac{T_{i, j-1}+T_{i, j}}{2} \cdot \frac{H_{i, j-1}^{\tau}-H_{i, j}^{\tau}}{\Delta y}-\frac{\Delta x}{T_{i, j}+T_{i, j+1}}}{2} \cdot \frac{H_{i, j}^{\tau}-H_{i, j+1}^{\tau}}{\Delta y}+\varepsilon= \\
& \mu \frac{H_{i, j}^{\tau+1}-H_{i, j}^{\tau}}{\Delta t} \\
& \frac{T_{i-1, j}+T_{i, j}}{2} \cdot \frac{H_{i-1, j}^{\tau}-H_{i, j}^{\tau}}{\Delta x^{2}}-\frac{T_{i, j}+T_{i+1, j}}{2} \\
& \cdot \frac{H_{i, j}^{\tau}-H_{i+1, j}^{\tau}}{\Delta x^{2}}+ \\
& +\frac{T_{i, j-1}+T_{i, j}}{2} \cdot \frac{H_{i, j-1}^{\tau}-H_{i, j}^{\tau}}{\Delta y^{2}}-\frac{T_{i, j}+T_{i, j+1}}{2} \cdot \frac{H_{i, j}^{\tau}-H_{i, j+1}^{\tau}}{\Delta y^{2}}+\varepsilon= \\
& \mu \frac{H_{i, j}^{\tau+1}-H_{i, j}^{\tau}}{\Delta t} .
\end{aligned}
$$

$j-1, j, j+1$ - пространственные индексы расчетных точек по координате $y$.

Остальные значения приведены выше.

Аналогично (11) записывается конечноразностная аппроксимация уравнения (10) по неявной схеме. Для задач по определению инфильтрационного питания явная и неявная схема равнозначны по математической точности, но с позиций гидрогеологии они разные. Явная схема характеризует инфильтрационное питание на начало расчетного периода, а неявная на его конец. Целесообразно выполнить расчеты по двум представленным схемам и в прямых прогнозных задачах использовать три варианта - два с рассчитанной величиной $\varepsilon$ и третий со средним значением. Решение эпигнозной задачи даст однозначный ответ, какая из рассчитанных величин максимально достоверна.

Анализ результатов. Все рассмотренные методы применимы для определения величина инфильтрационного питания на исследуемой территории по данным режимных наблюдений. Выполнены расчеты за период 1989 -1991 годы. Получены положительные и отрицательные значения.

Диапазон изменения положительных величин максимальные значения соответствуют апрелю 1989 года - 1,2 × 10 $0^{-5}$ м/сут, минимальные положительные результаты получены в октябре 1989 года $-5 \times 10^{-5} \mathrm{M} /$ сут.

Диапазон изменения отрицательных величин максимальными отрицательными значениями

\begin{tabular}{|c|c|c|c|c|c|c|c|c|}
\hline 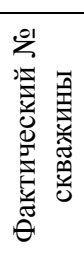 & 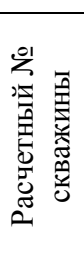 & 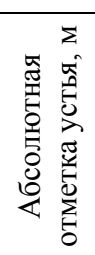 & 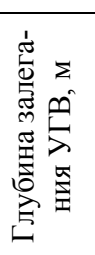 & 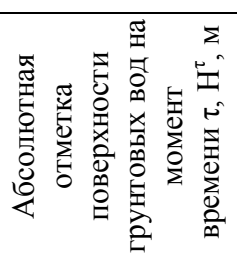 & 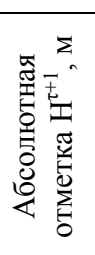 & 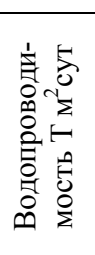 & 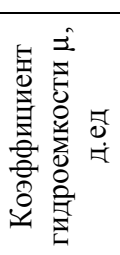 & 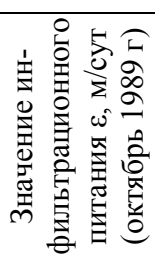 \\
\hline 166 & 1 & 123,5 & 6,19 & 6,36 & 6,19 & 1,24 & 0,012 & $-7,5 \times 10^{-5}$ \\
\hline 36 & 2 & 134,0 & 11,55 & 11,68 & 11,55 & 3,33 & 0,025 & $3,2 \times 10^{-5}$ \\
\hline 181 & 3 & 151,5 & 6,07 & 6,2 & 6,07 & 1,418 & 0,017 & $1,1 \times 10^{-5}$ \\
\hline
\end{tabular}
характеризуются май, июль и август 1989 года $1,9 \times 10^{-5}$ м/сут., а минимальные отрицательные величины получены в феврале 1989 года $-8 \times 10^{-5}$ м/сут.

Пример расчета величины $\varepsilon$ представлен в таблице. 1.

Таблица 1 Результаты расчета величины $\varepsilon$

Результаты расчета значения инфильтрационного питания в скважине 36 за период 1989 - 1991 гг. представлены на рисунке 2 . 


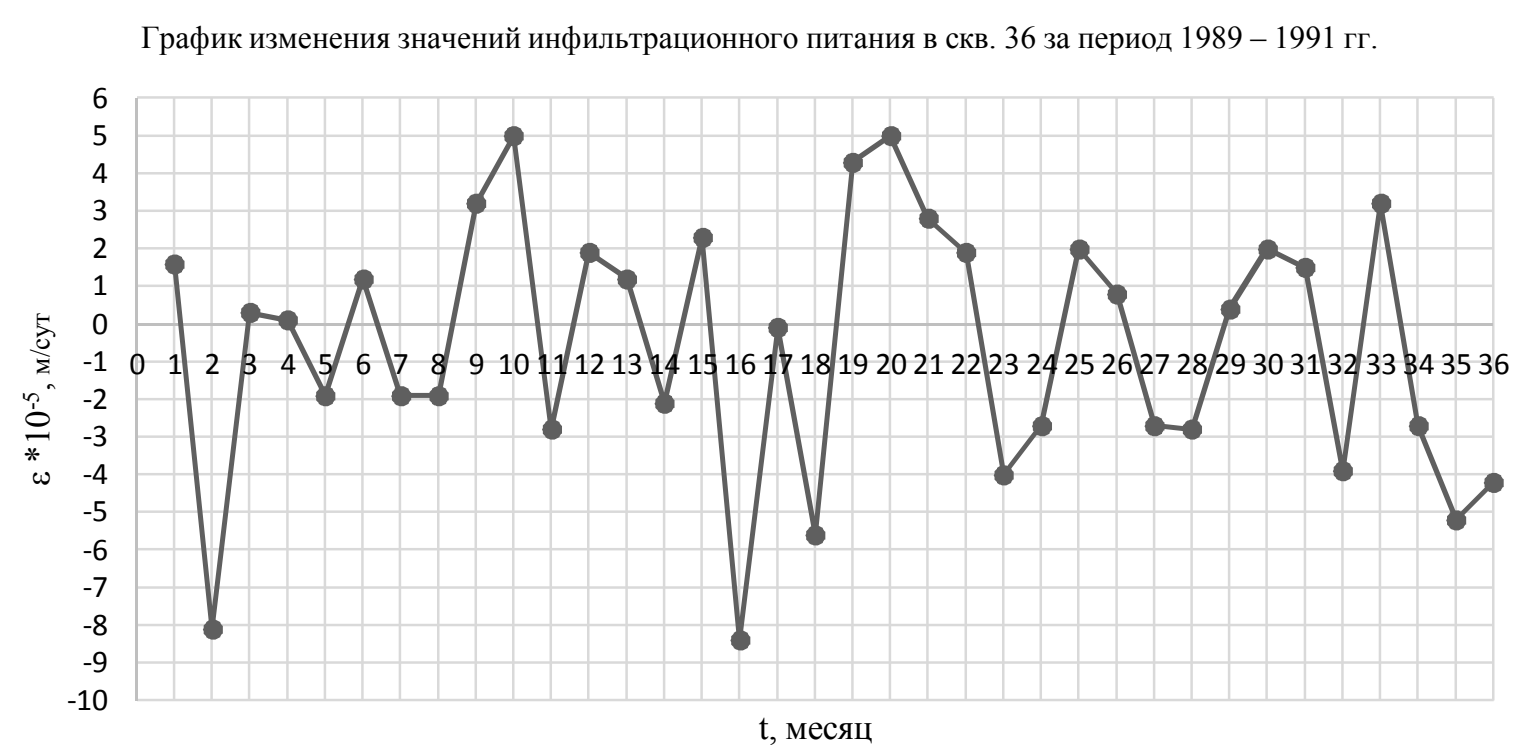

Рис. 2. Результаты расчета значений инфильтрационного питания в скважине 36

Выводы. 1. Изложенные методы приемлемы для решения поставленных задач без ограничений при наличии режимных наблюдений.

2. Эти методы хорошо согласуются друг с другом, что является подтверждением их высокой достоверности.

3. Инфильтрационное питание в разные годы характеризуется положительными и отрицательными значениями, но в многолетнем разрезе суммарная величина его положительная и составляет $1-1,5 \times 10^{-5}$ м/сут., что обеспечивает очень медленный подъем уровня грунтовых вод.

\section{Библиографические ссылки}

ShestakovV. M. 1979.Dinamika podzemnyh vod [The dynamics of ground water]. MGU Moskow (in Russian).
Sitnikov A. B., Golovchenko Ju. G., Tkachenko K. D. 2003.Gidrogeologicheskaja stancija "Feofanija": mnogoletnie issledovanija I rezul'taty [Hydrogeological station "Feofaniya": long-term research and results]. Kyiv (in Russian).

Rudakov V. K. 1970.Metody prognoznyh raschetov vlijanija oroshenija na rezhim gruntovyh vod [Methods for predicting the effect of irrigation on the groundwater regime]. Hydrogeological forecasts in connection with land irrigation and wather supply. Dnipro. (in Russian).

Zhernov I. Je., Soldak A. G., Kushh P. Ju., Gryza O. O. 1972. Melioratyvna gidrogeologija [Meliorative gidrogeologiya]. Vyshha shkola, Kyiv (in Ukranian). 